\title{
L'évaluation de la protection radiologique des travailleurs dans les centrales nucléaires
}

\author{
J. LOCHARD $(*)$, C. MACCIA $\left({ }^{*}\right)$ et P. PAGÈS $(* *)$ \\ (Manuscrit reçu le 5 mars 1981)
}

\begin{abstract}
RÉSUMÉ
La mise en œuvre du principe d'optimisation de la radioprotection des travailleurs dans les centrales nucléaires se pose en des termes différents de ceux habituellement retenus dans le cas du public. En effet, l'interdépendance entre les objectifs de production et de protection des travailleurs conduit à exprimer le coût d'une mesure de protection comme le bilan des dépenses (investissement+exploitation) directement liées à la mesure envisagée et des coûts de production éventuellement évités.

Une présentation formalisée permet d'expliciter les principaux paramètres nécessaires à l'évaluation du coût et de l'efficacité d'une mesure. Les conditions de définition et d'utilisation d'une valeur monétaire de l'homme-sievert issue d'une analyse $a$ posteriori des mesures de protection sont ensuite précisées.
\end{abstract}

\begin{abstract}
The methodological framework to put into practice the optimisation principle for occupational radiation protection in nuclear power plants differs from the procedure generally described for the protection of the public. The interdependence between production and protection objectives leads to expressing the cost of a protective action as the balance between expenditures (investment and operation) directly associated with the action and the potential operational costs saved.

The main parameters for evaluating the effectiveness of a dose reduction action are described. The way to deal with a monetary value of the man-sievert issued from an a posteriori analysis of a dose reduction action is discussed.
\end{abstract}

(*) C.E.P.N. (Centre d’étude sur l'évaluation de la protection dans le domaine nucléaire), B.P. 48,92260 Fontenay-aux-Roses.

(**) Commissariat à l'énergie atomique, I PS $\wedge$. Département de Protection. B P 6. 92260 Fontenay-aux-Roses

RADIOPROTECTION, VOL. $16-0033-8451 / 1981 / 201 / \$ 5.00 / \subset$ Bordas-Dunod. 


\section{PRÉSENTATION}

Le problème de la recherche des niveaux «ALARA » dans le cadre de la radioprotection des travailleurs des centrales nucléaires se pose en des termes différents de ceux habituellement retenus pour la protection du public dans le cas des rejets d'effluents radioactifs [1]. A la réduction des doses collectives délivrées au public correspond toujours une augmentation des dépenses d'investissement et/ou d'exploitation pour le producteur d'électricité. Et, d'une manière générale, plus le niveau de protection déjà atteint est grand, plus les coûts supplémentaires pour réduire le niveau de dose résiduel sont importants : les coûts de protection suivent donc la loi des rendements décroissants.

Dans le cas de la protection des travailleurs, une réduction de la dose collective ne conduit pas forcément à une augmentation concomitante des coûts pour l'exploitant. En d'autres termes, l'augmentation des coûts d'investissement et d'exploitation résultant directement de la mise en œuvre d'une mesure de protection peut être, dans certaines conditions, inférieure à la réduction des coûts d'exploitation que cette même mesure permet de réaliser par ailleurs.

Afın de rendre plus concret ce phénomène, il convient de décrire schématiquement comment les mesures prises pour réduire l'exposition des travailleurs dans une centrale interfèrent avec son exploitation. Dans les développements qui suivent, nous ne considérons que l'irradiation externe des travailleurs, la contamination interne par inhalation ne représentent dans les conditions normales d'exploitation qu'une fraction négligeable de l'exposition totale. En ce qui concerne l'irradiation externe, elle est, pour une population donnée de travailleurs, fonction du débit de dose au contact ou à proximité de certains composants de l'installation et du temps d'intervention sur ces composants (y compris les temps de circulation pour y accéder). Compte tenu des contraintes légales et/ou administratives concernant les limites de doses individuelles, il faut distinguer deux types de situation du point de vue de l'organisation du travail dans une centrale (qu'il s'agisse de la période de marche ou d'arrêt de la tranche).

Un premier type concerne les interventions sur des composants où le débit de dose est suffisamment faible pour que le travail puisse y être effectué dans des conditions normales, c'est-à-dire sans précaution particulière, par un ou plusieurs individus (selon la complexité de la tâche) sans considération de limites de temps et dans des conditions de productivité du travail moyennes. Bien que ces tâches contribuent à la dose collective, elles n'induisent pas de coûts supplémentaires d'exploitation par rapport à ceux que l'on rencontrerait dans une installation non nucléaire $\left({ }^{1}\right)$. Les mesures prises en vue de réduire les doses relatives à cette catégorie d'interventions se traduisent par des dépenses supplémentaires (d'investissement et éventuellement d'exploitation) et sont donc de même nature que les actions de protection pour le public.

( $\left.{ }^{1}\right)$ Cette affirmation n'est valable que si l'on néglige les coûts relatifs à la mise en place, à l'exploitation du système de surveillance radiologique et à la formation du personnel dans la centrale. 
Le deuxième type de situation concerne les interventions sur des composants ou dans des lieux où le débit de dose est tel que, compte tenu des limites individuelles, il convient d'adopter une forme d'organisation du travail moins productive: préparation des chantiers, multiplicité des équipes d'intervention, protection renforcée des travailleurs. Les temps d'intervention sont, dans ces conditions, la plupart du temps disproportionnés par rapport à ceux qui seraient nécessaires pour réaliser une tâche similaire en l'absence d'irradiation. De plus, certains des travaux effectués pendant l'arrêt de tranche interviennent sur le chemin critique et donc déterminent en partie la durée totale de l'arrêt et par conséquent son coût dans la mesure où ils entraînent une augmentation de l'indisponibilité de la tranche et donc le recours à une source d'énergie de remplacement. Notons, par ailleurs, que lorsqu'il s'agit des travaux d'entretien et de maintenance exécutés pendant l'arrêt, le personnel utilisé provient pour une partie d'entreprises extérieures et induit, de ce fait, des charges d'exploitation supplémentaires.

Cette deuxième catégorie d'interventions est donc à l'origine de surcoûts d'exploitation et toute mesure de protection qui vise à réduire d'une manière notable soit le débit de dose, soit le temps d'intervention, aura pour conséquence une diminution de ces coûts. L'ampleur de cette diminution ne semble pas négligeable. En l'absence de données françaises, on peut utiliser comme référence des exemples cités dans une étude américaine très détaillée entièrement consacrée à ce problème [2]. Ainsi, la réduction du débit de dose sur un composant où intervient du personnel d'entreprises extérieures conduit d'une part à une diminution de 0,17 homme-sievert de la dose collective, d'autre part à une économie de 12000 Sen ce qui concerne les coûts d'exploitation annuels. Un autre exemple encore plus significatif est relatif à une réduction du temps de travail pour une tâche sur le " chemin critique " grâce à un entraînement préalable sur maquette du personnel de l'entreprise extérieure concernée. Le coût d'exploitation annuel évité est alors estimé à 270000 \$, la réduction de la dose collective étant de 0,20 homme-sievert. Le montant des dépenses qui ont été engagées soit pour réduire le débit de dose soit pour construire la maquette n'est pas mentionné dans l'étude citée. Il est néanmoins certain que dans le deuxième cas les bénéfices (coûts d'exploitation évités) l'emportent largement sur les coûts (dépenses de protection) si l'on considère plusieurs années de fonctionnement de l'installation.

A la lumière de ces données, il convient donc de substituer à la notion classique de coût de protection telle qu'on l'envisage pour la protection du public par exemple, une structure de coûts plus complexe intégrant à la fois des éléments négatifs (les coûts additionnels des mesures) et des éléments positifs (les coûts d'exploitation évités). Il est à noter que ce phénomène n'est pas une particularité propre au domaine qui nous concerne, mais qu'on le rencontre pratiquement toujours en matière de prévention médicale [3]. En effet, aux coûts relatifs à la mise en place d'une politique de prévention d'une pathologie doivent être retranchés les coûts des soins évités du fait de l'efficacité de cette politique. Le coût d'une politique de prévention représente donc un bilan économique et non strictement une dépense. 
D’une manière plus générale, le problème soulevé ici est celui de l'interdépendance entre les fonctions de protection et de production propres à l'activité considérée. Ce problème méthodologique jusqu'à présent très rarement signalé mérite une attention particulière [4].

\section{LE MODÈLE}

L'intérêt d'une présentation formalisée du problème méthodologique présenté ci-dessus réside essentiellement dans l'explicitation des principaux paramètres nécessaires à l'évaluation des mesures de protection. Elle permet donc de repérer quel type d'information et quelles données sont indispensables pour passer à une phase ultérieure de quantification.

Le problème consiste à prendre en compte, de la manière la plus simple, les répercussions d'ordre économique et sanitaire consécutives à la mise en œuvre d'une mesure de protection pour les travailleurs. Soit $P_{0}$ le coût de production de l'électricité, $X_{0}$ le coût de protection et $S_{0}$ la dose collective aux travailleurs associée à un niveau de protection de référence noté $N_{0}$. L'impact d'une mesure $i$ conduit à des valeurs nouvelles $P_{i}, X_{i}$ et $S_{i}$, et les variations peuvent s'expliciter comme suit :

\subsection{Réduction de La dose Collective $\Delta S=S_{0}-S_{i}$}

La mesure $i$ est susceptible d'affecter un certain nombre de tâches. La dose collective associée à une tâche donnée $j$ s'exprime comme suit :

$$
S_{j}=d_{j} \times K_{j} \times t_{j}
$$

avec $d_{j}[\mathrm{rem} / \mathrm{h}]$, le débit de dose pour la tâche $j ; t_{j}[\mathrm{~h}]$, la durée de la tâche; $K_{j}$, l'effectif de travailleurs intervenant pour $j\left({ }^{2}\right)$ avec $K_{j} \geqq\left(t_{j} \times d_{j}\right) / L$, où $L$ est la limite administrative individuelle.

$$
\Delta S_{j}=K_{j} d_{j} \Delta t_{j}
$$

si la mesure porte sur la durée d'intervention;

$$
\Delta S_{j}=K_{j} t_{j} \Delta d_{j}
$$

si la mesure porte sur le débit de dose.

( ${ }^{2}$ ) On suppose ici que la tâche $j$ occupe un seul travailleur à la fois. Lorsque la tâche nécessite l'intervention simultanée de plusieurs travailleurs, il faut raisonner en termes d'équipe. 


\subsection{Augmentation des couts de protection $\Delta X=X_{i}-X_{0}$}

Le surcoût de protection associé à la mesure $i$ peut être évalué de deux manières selon l'horizon retenu :

$$
\Delta X=I_{x, i}+k^{\prime} E_{x, i}
$$

si l'on envisage le coût total, sur la durée de vie de l'i'ıstallation;

$$
\Delta X=k I_{x, i}+E_{x, i}
$$

si l'on cherche à déterminer le surcoût annuel, avec $I_{x, i}[\mathrm{~F}]$, coût d'investissement total de protection pour la mesure $i$ envisagée; $E_{x, i}[\mathrm{~F} / \mathrm{an}]$, coût annuel d'exploitation directement associé à la mesure $i$ de protection; $k^{\prime}$, coefficient d'actualisation; $k$, coefficient d'amortissement.

\subsection{VARIATION DU COÔT DE PRODUCTION $\Delta P=P_{i}-P_{0}$}

Le coût total actualisé de production est égal à $I_{p}+k^{\prime} E_{p}$ avec $I_{p}$, l'investissement total de la centrale (non compris les investissements de protection); $E_{p}$, le coût d'exploitation annuel (non compris les frais d'exploitation relatifs à l'entretien et la maintenance des mesures de protection.

Dans l'optique d'une évaluation annuelle, le coût de production est : $k I_{p}+E_{p}$.

On peut raisonnablement faire l'hypothèse que les mesures de protection n'affectent pas le niveau des autres investissements et donc que $\Delta I_{p}=0$. Quant à la variation des coûts d'exploitation, elle est égale à :

$$
\Delta E_{p}=c_{a} \Delta T_{a}+C_{i n} \Delta W_{i n}+C_{e x} \Delta W_{e x},
$$

avec $\Delta T_{a}[\mathrm{~h}]$, variation de la durée de l'arrêt pour rechargement; $c_{a}[\mathrm{~F} / \mathrm{h}]$, le coût de remplacement de l'électricité; $\Delta W_{i n}[\mathrm{~h}]$, variation de la quantité de travail effectué par le personnel interne à la centrale; $C_{i n}[\mathrm{~F} / \mathrm{h}]$, le coût moyen de l'heure d'agent interne; $\Delta W_{e x}[\mathrm{~h}]$, variation de la quantité de travail effectué par le personnel extérieur; $C_{e x}[\mathrm{~h}]$, le coût moyen de l'heure de l'agent extérieur.

\section{L'ANALYSE ÉCONOMIQUE DES MESURES DE PROTECTION}

Confronté au problème du choix parmi plusieurs mesures de protection envisageables, et en adoptant comme critère de sélection la recherche d'une allocation efficace des ressources de protection, il est possible, compte tenu des évaluations de $\Delta S, \Delta X$ et $\Delta P$ d'analyser et classer les différentes mesures selon leur rapport coût-efficacité.

Selon l'importance de la variation des coûts d'exploitation $\Delta E_{p}$ et surtout le sens de cette variation, on peut envisager deux catégories de mesures : celles pour lesquelles la somme des variations des coûts de protection et d'exploitation est positive $(\Delta X+\Delta P>0)$ et celles où cette somme est négative ou éventuellement nulle. Ce dernier cas se produit lorsque les dépenses de protection entraînées par 
la mise en œuvre d'une mesure sont inférieures (ou égales) aux coûts d'exploitation évités et dans ce cas $\Delta X+\Delta P \leqq 0$.

Si l'on considère un ensemble de mesures de protection indépendantes notées A, B, C, ... ces deux situations peuvent être représentées graphiquement, à partir d'un niveau de protection de référence pour lequel la dose collective est $S_{0}$ et le coût total associé est $X_{0}+P_{0}(f i g .1)$.

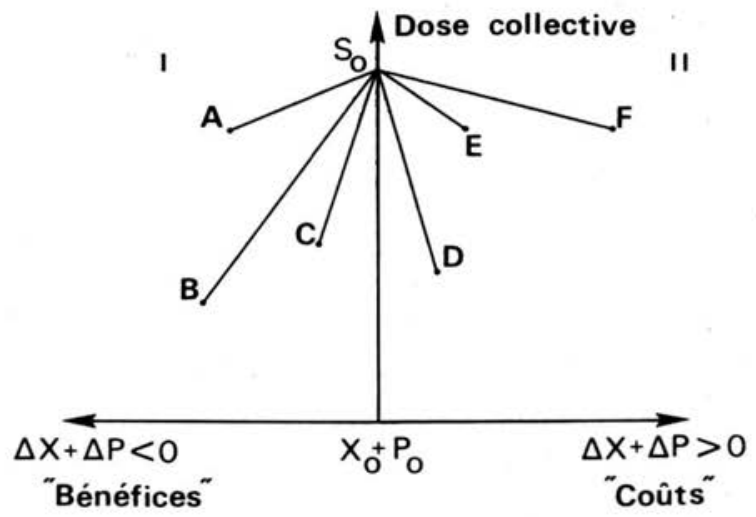

FIG. 1. - Exemple de "bilans » économiques pour un ensemble de mesures de protection.

Pour les options du cadran I qui présentent un bilan positif, la seule rationalité économique qui vise à réduire les coûts de production justifie leur adoption.

Pour les options du cadran II, la rationalité en terme d'efficience reprend ses droits. Le classement et la sélection des mesures dans le cadran s'effectue par comparaison des ratios coût-efficacité $\left({ }^{3}\right)$. Les résultats peuvent être visualisés à l'aide d'une courbe coût-efficacité. Elle est construite en portant en abscisse les coûts cumulés et en ordonnée les doses résiduelles à partir de la situation de référence. La première option sélectionnée est celle qui est la plus " coûtefficace " et chaque point suivant traduit la prise en compte de l'option la plus " coût-efficace " parmi les options restantes.

S'il existe soit une contrainte de budget, soit une contrainte sur la réduction de la dose collective, l'analyse coût-efficacité permet de déterminer les mesures devant être sélectionnées pour satisfaire les contraintes. La figure 2 illustre, par exemple, une courbe coût-efficacité pour des mesures du type de celle du cadran II de la figure 1.

L'approche coût-efficacité permet d'associer à chaque mesure un coût marginal de l'unité de dose évitée. L'interprétation classique est de considérer le ratio coût-efficacité de la dernière mesure adoptée comme la valeur monétaire

$\left({ }^{3}\right)$ Pour une présentation plus détaillée de l'analyse coût-efficacité voir la référence [1]. 
implicitement accordée à l'homme-sievert. En l'absence d'une valeur de référence du coût de l'unité de dose (soit légale, soit administrative), l'analyse des actions passées de protection permet donc de déterminer quelle a été la valeur implicitement accordée à l'homme-sievert. Elle peut constituer un guide pour la politique de protection à venir dans la mesure où l'on cherche à garder, dans les

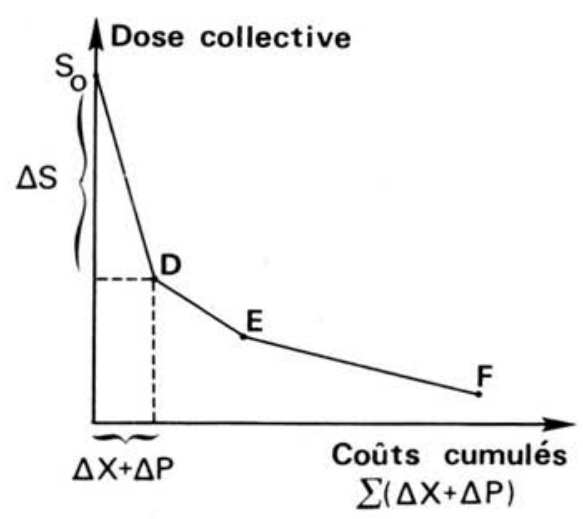

Fig. 2. - Analyse coût-efficacité des options D, E, F de la figure 1.

efforts de protection, une certaine cohérence avec les actions passées. Par ailleurs, à partir de la valeur monétaire de l'homme-sievert, on peut évaluer le prix implicitement accordé à la vie humaine en utilisant le coefficient dose-risque admis dans le domaine radiologique. Le passage à la valeur implicite de la vie humaine permet la comparaison avec d'autres activités industrielles génératrices de risques pour lesquelles sont entrepris des efforts de protection $\left({ }^{4}\right)$.

\section{CONCLUSION}

L'hypothèse d'indépendance entre les niveaux de protection et les contraintes de production admise très largement dans le cas du public n'est pas légitime dans le cas de la protection radiologique des travailleurs. Le modèle développé ci-dessus vise à rendre compte de cette interdépendance en ce qui concerne les mesures de protection susceptibles de réduire la dose collective dans - les centrales nucléaires.

La principale conséquence de cette reformulation a trait à la signification que l'on peut accorder pour la détermination des niveaux de protection acceptables au coût marginal de l'unité de dose évitée ou, ce qui est équivalent,

$\left({ }^{4}\right)$ Cette comparaison n'est pas aisée lorsqu'il s'agit de risques diffus et qu'il n'existe pas, comme dans le domaine radiologique, de relations dose-risques sur lesquelles on constate un certain consensus. 
au coût implicite de la vie humaine. En effet, les rapports "dépenses de protection/diminution des risques » ne relèvent plus seulement d'une rationalité sanitaire ou de protection. La démarche qui consiste à utiliser les valeurs implicites de la vie humaine pour comparer et éventuellement gérer des risques d'origine diverses, bien que largement pratiquée [4 et 5], doit cependant être maniée avec prudence et se fonder sur une analyse préalable de la structure des coûts.

Cet article constitue la première partie d'une étude menée au C.E.P.N. Une application de la méthode présentée ici sera développée dans une publication ultérieure.

\section{BIBLIOGRAPHIE}

[1] LOChARD J., MACCIA C., PAGES P. Méthode pour la mise en cuvre du principe d'optimisation de la radioprotection proposée par la C.I.P.R. Rapport CEA-R-5071, 1980.

[2] Pelletier C., Vollleque G. Potential benefits of reducing occupational radiation exposure. Report AIF/NESP-010R, 1979.

[3] Levy E., Bungener M., Dumenil G., FAGnAni F. Évaluer le coût de la maladie, Paris : Dunod, 1977.

[4] LOCHARD J. The optimisation of radiation protection, an analysis of the cost-benefit model proposed by the International Commission on Radiological Protection. Nucl. Safety, 1981, 22, (sous presse).

[5] SIDAll E. Nuclear safety in perspective. 19. Anmual conference of the Canadian nuclear association, Toronto, 1979.

[6] O'DONNEL E. P. What price safety? A probabilistic cost-benefit evaluation of existing engineered safety features. In: Nuclear power reactor safety, E.N.S./A.N.S. Topical Meeting, Brussels, 1978. 